\title{
TCP10L negatively regulates alpha-fetoprotein expression in hepatocellular carcinoma
}

\author{
Suqin Shen ${ }^{\#}$, Huan Feng ${ }^{\#}$, Longjiang Liu, Wei Su, Long Yu ${ }^{*} \mathcal{E}$ Jiaxue Wu \\ The State Key Laboratory of Genetic Engineering, Zhongshan Hospital and School of Life Science, Fudan University, Shanghai 200438, \\ China
}

\begin{abstract}
Alpha-fetoprotein (AFP) is one of the most commonly used and reliable biomarkers for Hepatocellular carcinoma (HCC). However, the underlying mechanism of AFP expression in HCC is poorly understood. In this study, we found that TCP10L, a gene specifically expressed in the liver, is down-regulated in HCC and that its expression inversely correlates with AFP expression. Moreover, overexpression of TCP10L suppresses AFP expression whereas knockdown of TCP10L increases AFP expression, suggesting that $\mathrm{TCP} 10 \mathrm{~L}$ might be a negative regulator of AFP. We found that TCP10L is associated with the AFP promoter and inhibits AFP promoter-driven transcriptional activity. Taken together, these results indicate that TCP10L negatively regulates AFP expression in HCC and that it could be a potential prognostic marker and therapeutic target for HCC. [BMB Reports 2020; 53(8): 431-436]
\end{abstract}

\section{INTRODUCTION}

Hepatocellular carcinoma (HCC) is the fourth leading cause of cancer-related deaths, ranking sixth among new cases worldwide (1). A more terrible feature of hepatocellular carcinoma is that it lacks typical symptoms in the early stage. Most patients have reached an advanced stage when at initial diagnosis, and the surgical treatment including partial liver resection and liver transplantation are not satisfactory $(2,3)$. In addition, the high incidence of recurrence and metastasis compromises the longterm therapeutic effect of surgical treatment, which makes the early diagnosis and treatment of this tumor ineffective (4). There-

*Corresponding authors. Long Yu, Tel: +86-31246554; Fax: +8631246553; E-mail: longyu@fudan.edu.cn; Jiaxue Wu, Tel: +86-31 246554; Fax: +86-31246553; E-mail: jiaxue@fudan.edu.cn

${ }^{\#}$ These authors contributed equally to this work.

https://doi.org/10.5483/BMBRep.2020.53.8.008

Received 10 January 2020, Revised 7 February 2020, Accepted 25 February 2020

Keywords: Alpha-fetoprotein (AFP), Hepatocellular carcinoma (HCC), T-complex protein 10A homolog 2 (TCP10L), Transcriptional regulation fore, it is critical to elucidate the molecular mechanisms underlying HCC development and find potential novel molecular targets thus developing new strategies for HCC treatment.

Alfa-fetoprotein (AFP) is a polypeptide of approximately 600 amino acids and was first detected by electrophoresis in human fetal serum. AFP is expressed at high levels in the fetal liver and visceral endoderm of the yolk sac (5). Shortly after birth, the AFP gene is dramatically repressed and is normally expressed at extremely low levels in the adult liver. However, AFP can be reactivated during liver regeneration and hepatocellular carcinogenesis. Importantly, AFP is a secretory protein and changes in its blood level make it an important marker for HCC diagnosis and patient prognosis that is widely used in clinical practice. However, the underlying mechanism of AFP re-expression in HCC remains largely unknown.

Five distinct regulatory elements that govern AFP expression are found within $7.6 \mathrm{~kb}$ of DNA upstream of the AFP transcription start site: a 250-bp tissue-specific promoter, a repressor domain which lies within $1 \mathrm{~kb}$ of the AFP transcription start site that is at least partially responsible for the decrease in AFP gene expression in the adult liver, and three independent enhancers located 2.5, 5.0, and $6.5 \mathrm{~kb}$ upstream of the AFP promoter that are essential for AFP transcription in vivo and continue to be active in the adult liver (6). Several known liverenriched factors that positively or negatively regulate AFP transcription have been identified. Hepatocyte nuclear factors (HNFs) nuclear factor 1 (NF-1) and CAAT/enhancer binding protein (C/ $\mathrm{EBP})$, which mediate the transcription of most of the liver-specific genes, have been shown to bind to a region at 120 from the transcription start site $(+1)$ promoter and are capable of activating AFP gene transcription both in vitro and in vivo $(7,8)$. Likewise, transcriptional repressors involved in postnatal AFP silencing have been identified. For example, zinc finger and BTB domain containing 20 (ZBTB20) directly binds to a region of the AFP promoter between $-104 /-86$ and downregulates the activity of the AFP promoter $(9,10)$. Zinc-fingers and homeoboxes 2 (ZHX2) suppresses the activity of HNF1 transcription factors at the level of the AFP promoter (11). p53 mediates AFP repression by competing with HNF3 for binding to DNA in the repressor region $-838 /-250$ of the AFP gene and altering its chromatin structure $(12,13)$. Therefore, postnatal repression of AFP transcription may involve the combinatorial action

ISSN: 1976-670X (electronic edition)

Copyright (C) 2020 by the The Korean Society for Biochemistry and Molecular Biology

cc This is an open-access article distributed under the terms of the Creative Commons Attribution Non-Commercial License (http://creativecommons.org/licenses/by-nc/4.0) which permits unrestricted non-commercial use, distribution, and reproduction in any medium, provided the original work is properly cited. 
of multiple distinct repressors. In summary, the physiological mechanism of AFP repression in the postpartum liver is not well understood, including the involved trans-acting repressors and the corresponding cis-acting elements.

T-complex protein 10A homolog 2 (TCP10L) was first cloned by Chen Z. et al. in 2003 through a differentially displayed hybrid from a human liver cDNA library. It is specifically expressed in the liver and testis in normal human tissues (14, 15). TCP10L contains a putative leucine zipper (LZ) domain, which is required for homodimerization and heterodimerization with other LZ-containing proteins (16-19). Recently, we reported that TCP10L is a potential tumor suppressor in HCC (20). TCP10L stabilizes the MAD1 protein level through direct interaction, and they cooperatively regulate cell cycle progression (19).

In this study, we aim to explain the relationship between AFP and TCP10L in clinical HCC samples, and to discover the molecular mechanism of how TCP10L regulates AFP by using liver cancer cell lines. Due to the important role of AFP in liver cancer diagnosis, the study of TCP10L will provide some new evidence and support for the clinical diagnosis and treatment of liver cancer.

\section{RESULTS}

TCP10L expression is negatively associated with AFP level Previously, we found that the TCP10L expression level is downregulated in HCC (20). To understand the clinical significance of this finding, we further analyzed the relationship between the expression level of TCP10L mRNA and clinical pathological features of HCC. Based on the expression level of TCP10L in their HCC tissues, 102 patients were divided into two groups: low TCP10L expression and moderate or high TCP $10 \mathrm{~L}$ expression. As shown in Table 1, low expression of TCP10L significantly correlated with high serum AFP values $(P=0.024)$. No other correlations were found between TCP10L expression and other clinical pathological features, suggesting that TCP10L expression may negatively correlate with the AFP level.

To further confirm the relationship between the expression levels of TCP10L and AFP in HCC, we analyzed TCP10L and AFP mRNA expression in paired tissues from another $106 \mathrm{HCC}$ patients using quantitative real-time PCR (qRT-PCR). As shown in Fig. $1 \mathrm{~A}$ and $1 \mathrm{~B}, \mathrm{TCP} 10 \mathrm{~L}$ was significantly downregulated in $50 \%$ of the HCC specimens compared to the corresponding control tissues (53 of 106 cases showed a more than two-fold decrease in TCP10L mRNA compared to the normal adjacent liver tissue). Furthermore, the expression level of AFP was dramatically increased in most of the HCC tissues, which is consistent with previous results. Importantly, statistical analysis indicated that TCP10L expression negatively correlated with AFP expression (Pearson correlation coefficient, $r=-0.2198$, $\mathrm{P}<0.001$ ) (Fig. 1C). Interestingly, a guiding dotted line in Fig. 1C indicated that low TCP10L expression was significantly negatively correlated with AFP expression (Pearson correlation coefficient, $r=-0.5594, \mathrm{P}<0.001)$. Moreover, the protein levels of TCP10L and AFP in 12 paired HCC specimens also displayed a significant inverse correlation as determined by western blot (Fig. 1D). These results indicate that the expression level of TCP10L negatively correlates with the AFP level in $\mathrm{HCC}$, suggesting that TCP10L may be a negative regulator of AFP.

\section{TCP10L suppresses AFP expression in HCC cells}

To investigate the potential functional relationship between TCP10L and AFP, we generated stably-overexpressed Flag-tagged TCP10L in Hep3B cells which expressed AFP at a high level and investigated the expression of AFP by western blot, ELISA and qRT-PCR. As shown in Fig. 2A, the protein level of AFP dramatically decreased in stably-overexpressed Flag-tagged TCP10L Hep3B cells compared to the control cells. The TCP10L protein contains a putative leucine zipper (LZ) domain, which is required for it to suppress gene transcription (15). Next, we generated the stably-overexpressed leucine zipper domain deleted mutant TCP10L- $\Delta \mathrm{LZ}$ and examined the AFP expression level. As shown in Fig. 2A, TCP10L- $\Delta \mathrm{LZ}$ overexpression failed to inhibit AFP expression in Hep3B cells, suggesting that TCP10L suppresses AFP expression through its leucine zipper domain. The AFP level in cell culture medium was also examined in control, TCP10L overexpressed and TCP10L- $\Delta \mathrm{LZ}$ overexpressed Hep3B cells by ELISA. As shown in Fig. 2B, compared to the control and TCP10L- $\Delta \mathrm{LZ}$ overexpressed Hep3B cells, the AFP level in TCP10L overexpressed cell culture medium was dramatically reduced. AFP mRNA levels were then examined in control, TCP10L overexpressed and TCP10L- $\Delta \mathrm{LZ}$ overexpressed Hep3B cells by qRT-PCR. As shown in Fig. 2C, compared to the control and TCP10L- $\Delta \mathrm{LZ}$ overexpressed Hep3B cells, the AFP mRNA level in TCP10L overexpressed cells was also dramatically reduced. Taken together, these results indicate that TCP10L suppresses AFP expression in HCC cells through its leucine zipper domain.

Next, we knocked down the expression of TCP10L using shRNA in HCC cells and examined the AFP level. A lentiviral vector expressing the TCP10L shRNA construct (named LentiS3) and a control vector containing a non-targeting sequence (named Lenti-NS) were transfected into Hep3B and HepG2 cells. As shown in Fig. 2D, compared to the control cells, the TCP10L protein was efficiently suppressed in TCP10L shRNA infected Hep3B and HepG2 cells. In contrast, the AFP protein dramatically increased in TCP10L shRNA infected Hep3B and HepG2 cells compared to the control cells as determined by western blot. Furthermore, compared to the control cells, the AFP level in the culture medium of TCP10L shRNA infected Hep3B and HepG2 cells was also dramatically increased as determined by ELISA (Fig. 2E). Next, AFP mRNA levels were examined in control and TCP10L shRNA infected Hep3B and HepG2 cells by qRT-PCR. As shown in Fig. 2F, compared to the control shRNA infected Hep3B and HepG2 cells, the AFP mRNA level in TCP10L shRNA infected cells was also dramatically increased. These results indicate that downregulation of TCP10L enhances the expression of AFP. 
Table 1. Correlation of the clinic-pathological findings with TCP10L Downexpression in 102 cases of Human HCC

\begin{tabular}{|c|c|c|c|c|}
\hline \multirow{2}{*}{$\begin{array}{l}\text { Pathological } \\
\text { characteristic }\end{array}$} & \multicolumn{3}{|c|}{ TCP10L expression (T/N) } & \multirow{2}{*}{$P$ value } \\
\hline & Down-regulation (no. of case) & Up-regulation (no. of case) & Down-regulation proportion (\%) & \\
\hline \multicolumn{5}{|l|}{ Sex } \\
\hline Male & 50 & 31 & $61.73 \%(50 / 81)$ & 0.677 \\
\hline Female & 14 & 7 & $66.67 \%(14 / 21)$ & \\
\hline \multicolumn{5}{|l|}{ Age (years) } \\
\hline$\leq 50$ & 21 & 10 & $67.74 \%(21 / 31)$ & 0.490 \\
\hline$>50$ & 43 & 28 & $60.56 \%(43 / 71)$ & \\
\hline \multicolumn{5}{|l|}{ Family History } \\
\hline$(+)$ & 3 & 1 & $75.00 \%(3 / 4)$ & 0.605 \\
\hline$(-)$ & 61 & 37 & $62.24 \%(61 / 98)$ & \\
\hline \multicolumn{5}{|l|}{ Hepatitis History } \\
\hline$(-)$ & 29 & 13 & $69.05 \%(29 / 42)$ & 0.271 \\
\hline$(+)$ & 35 & 25 & $58.33 \%(35 / 60)$ & \\
\hline \multicolumn{5}{|l|}{$\mathrm{HBsAg}$} \\
\hline Negative & 16 & 9 & $64.00 \%(16 / 25)$ & 0.847 \\
\hline Positive & 47 & 29 & $61.84 \%(47 / 76)$ & \\
\hline \multicolumn{5}{|l|}{ AFP } \\
\hline Negative ( $\leq 20 \mathrm{ng} / \mathrm{ml})$ & 24 & 23 & $51.06 \%(24 / 47)$ & $0.024^{*}$ \\
\hline Positive (>20 ng/ml) & 40 & 15 & $72.73 \%(40 / 55)$ & \\
\hline \multicolumn{5}{|l|}{ Tumor Number } \\
\hline (single) & 52 & 33 & $61.18 \%(52 / 85)$ & 0.464 \\
\hline (multiple) & 12 & 5 & $70.59 \%(12 / 17)$ & \\
\hline \multicolumn{5}{|l|}{ Tumor Size $(\Phi, \mathrm{cm})$} \\
\hline$\leq 5$ & 27 & 21 & $56.25 \%(27 / 48)$ & 0.201 \\
\hline$>5$ & 37 & 17 & $68.52 \%(37 / 54)$ & \\
\hline \multicolumn{5}{|l|}{ Tumor Encapsulation } \\
\hline Absent & 39 & 18 & $68.42 \%(39 / 57)$ & 0.182 \\
\hline Present & 25 & 20 & $55.56 \%(25 / 45)$ & \\
\hline \multicolumn{5}{|c|}{ Portal Vein Tumor Thrombus } \\
\hline No & 36 & 26 & $58.06 \%(36 / 62)$ & 0.259 \\
\hline Yes & 27 & 12 & $69.23 \%(27 / 39)$ & \\
\hline \multicolumn{5}{|c|}{ Pathological Differentiation } \\
\hline I-II & 45 & 29 & $60.81 \%(45 / 74)$ & 0.511 \\
\hline III-IV & 19 & 9 & $67.86 \%(19 / 28)$ & \\
\hline \multicolumn{5}{|l|}{ TNM Clinical Stage } \\
\hline I & 29 & 18 & $61.70 \%(29 / 47)$ & 0.746 \\
\hline II-III & 35 & 19 & $64.81 \%(35 / 54)$ & \\
\hline \multicolumn{5}{|l|}{ Survival (d) } \\
\hline$\leq 1000$ & 24 & 14 & $63.16 \%(24 / 38)$ & 0.537 \\
\hline$>1000$ & 15 & 12 & $55.56 \%(15 / 27)$ & \\
\hline
\end{tabular}

Pearson Chi-square test was used. *Represents P values with signidifference. HCC, hepatocellular carcinoma.

\section{TCP10L suppresses AFP transcription through association with the AFP promoter}

Previously, we reported that TCP10L is located in the nucleus and functions as a transcriptional repressor (21); therefore, we assessed whether TCP10L represses transcription of AFP. The promoter region $(-2069 \sim+27 \mathrm{bp})$ of the human AFP gene was cloned from human genomic DNA and inserted into the
pGL3-basic vector to detect AFP transcription using the luciferase assay. As shown in Fig. 3A, compared to the control cells and cells with TCP10L- $\Delta \mathrm{LZ}$ overexpression, the transcriptional activity of the AFP promoter dramatically decreased in cells with TCP10L overexpression. Moreover, the inhibition of AFP promoter driven transcriptional activity by TCP10L was dose dependent (Fig. 3B), suggesting that TCP10L might repress the 


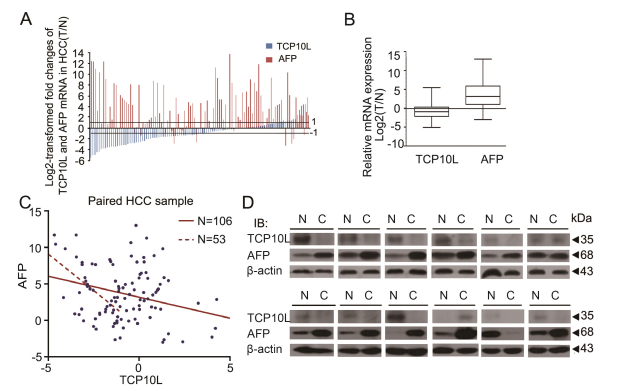

Fig. 1. The expression of AFP and TCP10L are negatively correlated in HCC. (A, B) The mRNA levels of AFP and TCP10L were analyzed in 106 HCC samples together with their corresponding non-cancerous tissues by quantitative RT-PCR. The columns show the fold change of AFP (red) and TCP10L (blue) mRNA levels in HCC samples compared to the corresponding adjacent noncancerous liver tissue, with beta-2-microglobulin serving as an internal control. (C) The scatter plot shows the correlation of TCP10L and AFP expression. The data was analyzed using a paired f-test, and correlation analysis was performed using Pearson's correlation test. The red line represents the correlation in 106 pairs of HCC samples. A guiding dotted line indicates the correlation between AFP and low TCP10L expression in 53 pairs of HCC samples. (D) The protein levels of AFP and TCP10L were analyzed in 12 HCC samples together with their corresponding non-cancerous tissues by western blot using the indicated antibodies, with $\beta$-actin serving as the loading control.

activity of the AFP promoter through its leucine zipper domain.

Next, TCP10L response regions in the AFP promoter were mapped. Two AFP mutants, namely, P1 (-2069 -1069 bp) and P2 $(-1069 \sim+27$ bp), were constructed (Fig. 3C) and subsequently co-transfected into Hep3B cells with pCMV-MycTCP10L. The luciferase reporter assay indicated that overexpression of TCP10L decreased the activity of the luciferase reporter harboring the P2 $(-1069 \sim+27 \mathrm{bp})$, but not the P1 $(-2069 \sim$ -1069 bp) mutant (Fig. 3D), suggesting that the TCP10L response region might be located in the -1069 to 27 region of the AFP promoter. We then tested whether TCP10L directly associates with the AFP promoter region using the ChIP assay. Hep3B cells that stably expressed exogenous Flag-TCP10L were lysed and immunoprecipitated using an anti-Flag antibody or an IgG control. The nucleotide sequence of the -981 to +12 nt region of the AFP promoter is shown in Fig. 3E and various length fragments (160 bp, $176 \mathrm{bp}, 243 \mathrm{bp}, 166 \mathrm{bp}$, $168 \mathrm{bp}$, and $193 \mathrm{bp})$ of AFP P $(-981 /+12)$ were amplified using the underlined primers. As shown in Fig. 3F, nt $-981 /-821$ of AFP was amplified in the samples pulled down with Flag antibodies but not with the IgG control by RT-PCR. We did not detect any PCR products for the other DNA fragments. ChIPquantitative polymerase chain reaction (qPCR) confirmed that endogenous TCP10L was enriched around this fragment (Fig. 3G). These data together demonstrate that TCP10L binds to the AFP promoter and that the responsive region is located between 981 bp and 821 bp upstream of the ATG start codon of AFP.

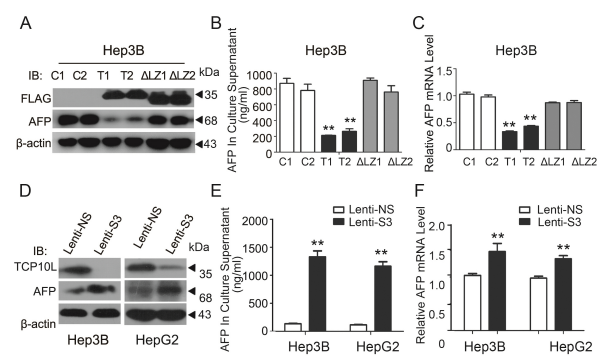

Fig. 2. TCP10L suppresses AFP expression in HCC cells. (A) The protein level of AFP in Hep3B cells stably expressing FLAG-TCP10L (T1, T2) and FLAG-TCP10L- $\Delta \mathrm{LZ}(\Delta \mathrm{LZ1}, \Delta \mathrm{LZ2})$ and in control cells $(\mathrm{C} 1, \mathrm{C} 2)$ was examined by western blot using the indicated antibodies, with $\beta$-actin serving as the loading control. (B) The AFP level in media was measured by ELISA. The mean values $\left( \pm\right.$ S.D.) are shown. ${ }^{* * P}<0.01$. (C) The mRNA level of AFP was measured by qRT-PCR. The mRNA level of $\beta 2-M G$ was used for normalization. The mean values $( \pm$ S.D.) are shown. $* * P<0.01$. (D) The protein levels of AFP and TCP10L in TCP10L knockdown (Lenti-S3) and control cells (Lenti-NS) were examined by western blot using the indicated antibodies. $\beta$-actin was used as a loading control. (E) The AFP level in media was measured by ELISA. The mean values $( \pm$ S.D.) are shown. $* * P$ $<$ 0.01. (F) The mRNA level of AFP in TCP10L knockdown (Lenti-S3) and control cells (Lenti-NS) was measured by qRT-PCR. The mRNA level of $\beta 2-M G$ was used for normalization. The mean values $( \pm$ S.D.) are shown. $* * P<0.01$.

\section{DISCUSSION}

In the present study, we found that TCP10L associates with the promoter region of AFP and suppresses its expression. Moreover, TCP10L is downregulated in HCC, and the expression of TCP $10 \mathrm{~L}$ is negatively correlated with AFP, suggesting that TCP10L might negatively regulate AFP expression in HCC and play an important role in HCC development.

Transcription repressors play important roles in tissue-specific and temporal gene expression $(22,23)$. Our previous results indicate that TCP10L is a transcription inhibitor and that it is expressed exclusively in the liver and testis. In this work, we found that TCP10L represses the activity of the AFP promoter and that this repression depends on the leucine zipper domain of TCP10L. These results provide the first direct evidence that TCP10L acts on the AFP promoter and negatively regulates AFP expression.

The AFP promoter contains binding sites for ubiquitous and tissue-specific transcription factors, such as HNF-1/NF-1, nkx2.8, fetoprotein transcription factor (FTF) and FoxA. There is a DNA binding site located at -135 of the AFP promoter for transcriptional repressor COUP-TF $(24,25)$. Additionally, p53 mediates AFP repression by competing with HNF3 for DNA binding in the repressor region of the AFP gene $(-838$ to -250$)$. The region between -1010 and -838 is required for Afr2-regulated AFP expression during liver regeneration $(26,27)$. Our results show that TCP10L interacts with the AFP promoter $(-981$ to -821$)$ and represses AFP gene transcription. Therefore, we hypothesize 


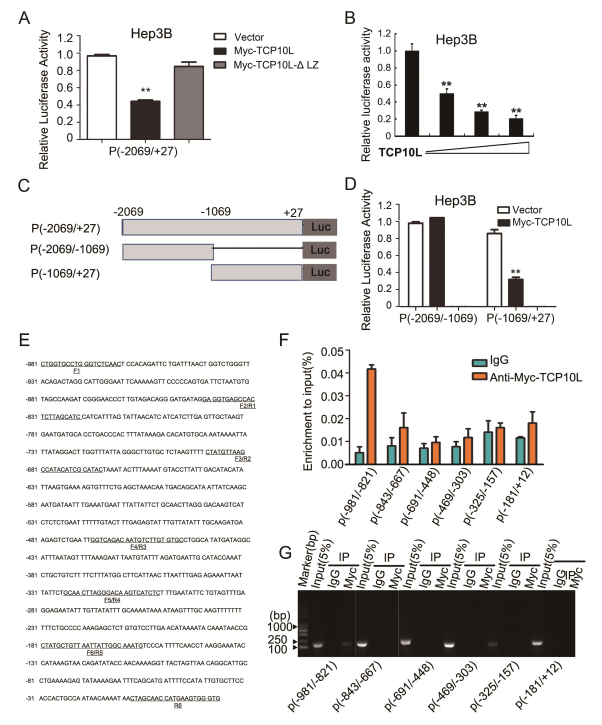

Fig. 3. TCP10L associates with the AFP promoter and inhibits its transcriptional activity. (A) TCP10 suppressed the transcriptional activity of the AFP promoter. Control vector, Myc-TCP10L or Myc-TCP10L$\triangle \mathrm{LZ}$ were co-transfected with the AFP full-length promoter (PGL3AFP) into Hep3B cells, and the transcriptional activity of the AFP promoter was examined by luciferase reporter assays. Reporter activity values are expressed as the mean \pm S.D., $* * P<0.01$. (B) Relative reporter activities were analyzed in Hep3B cells co-transfected with the AFP promoter and different dosages of Myc-TCP10L. Reporter activity values are expressed as the mean \pm S.D., $* * P<0.01$. (C) Schematic view of the luciferase reporter constructs containing various regions of the 5'-flanking region of the AFP gene. (D) Hep3B cells were transfected with Myc-TCP10L and the AFP reporter constructs as indicated. Reporter activity values are expressed as the mean \pm S.D., ${ }^{* * P}<0.01$. (E) The nucleotide sequences of the $5^{\prime}$ flanking region $(-981 /+12)$ of the AFP gene and the primers F1-F6 and R1-R6 used in ChIP-PCR and ChIP-qRT-PCR analysis are underlined. ( $F, G)$ TCP10L associated with the $-981 /-821$ region of the AFP promoter. TCP10L promoter regions were pulled down by chromatin immunoprecipitation using the indicated antibodies, followed by real time PCR (F) or PCR $(G)$ using the indicated primers.

that TCP10L might interact with other transcription factors to exert its biological effects. Through a typical leucine zipper (ZIP) motif, TCP10L could form homodimers or heterodimers with other ZIP motif-containing proteins (15). Our data shows that TCP10L represses the activity of the AFP promoter via the leucine zipper domain. We hypothesize that TCP10L might repress gene transcription via heterodimerization with other ZIP motif-containing proteins. Nevertheless, future studies are necessary to investigate whether other cofactors are also involved in this transcriptional repression. Overall, these results emphasize the complexity of AFP repression and provide further evidence that postnatal AFP shut-off requires many transcription factors.

The relationship between liver cell proliferation and AFP gene transcription has been noted but is not yet fully explored (28, 29). Postnatal AFP repression in the liver is coincident with the cessation of hepatocyte cell division, and AFP reactivation is often related to hepatocyte proliferation (30). Thus, it would be interesting to determine whether AFP reactivation in $\mathrm{HCC}$ reflects decreased TCP10L levels or activity. Whether AFP re-expression in TCP10L-knockdown cells leads to enhanced susceptibility for hepatocellular carcinogenesis remains under investigation. Our studies provide a better understanding of the function and regulatory role of TCP10L in HCC development and may also provide new targets for drug development.

\section{MATERIALS AND METHODS}

Information of "Generation of stable cell lines" is included in Supplementary Data.

\section{HCC specimens and cell lines}

Primary HCC and the corresponding non-tumorous liver tissues were freshly collected from HCC patients who underwent a hepatectomy at the Zhongshan Hospital (Shanghai, China). Samples were snap-frozen in liquid nitrogen immediately after surgery and stored at $-80^{\circ} \mathrm{C}$ until further use. The human HCC cell lines Hep3B (AFP positive) and HepG2 (AFP positive) were cultured in Dulbecco's Modified Eagle Medium (Gibco BRL, USA) supplemented with $10 \%(\mathrm{v} / \mathrm{v})$ fetal bovine serum (Gibco, USA) and $100 \mathrm{U} / \mathrm{ml}$ of penicillin at $37^{\circ} \mathrm{C}$ with $5 \%$ carbon dioxide.

\section{Luciferase reporter assays}

In a 24-well plate, Hep3B cells were co-transfected with pGL3 containing the AFP promoter, Renilla luciferase plasmid and the TCP10L expression plasmid or an empty vector control using the Lipofectamine 2000 reagent (Invitrogen, USA). Cells were harvested $30 \mathrm{~h}$ after transfection. Relative luciferase activity was determined using the Dual-Luciferase Reporter Assay System according to the manufacturer's instructions (Promega, USA). Experiments were repeated three times. The relative light units were measured by a luminometer (Promega, USA). The results are representative of the three independent experiments.

\section{Chromatin Immunoprecipitation (ChIP) Assay}

Physical associations between mammalian TCP10L and the AFP promoter were analyzed using a ChIP assay kit (Millipore, USA). The in vivo ChIP assay was performed using Hep3B cells. Cross linked protein-DNA complexes were immunoprecipitated with Flag antibodies. Isolated DNA was subjected to RT-PCR and analyzed by performing agarose gel electrophoresis, staining with ethidium bromide, and then visualizing using ultraviolet (UV) light. DNA fragments were recovered by phenol/ chloroform extraction and amplified through qRT-PCR. The primers used were listed in Supplementary Table 1.

Other materials and methods are listed in the supplementary materials and methods. 


\section{ACKNOWLEDGEMENTS}

This work was supported by the National Natural Science Foundation of China (81972712 to J. W., 81702742 to S.S., 31671263 to W. S.). We thank Yongjun Dang at the School of Basic Medical Science, Fudan University for fruitful scientific discussions and sharing of reagents. We thank Xing Gu at the Shanghai Eastern Hepatobiliary Surgery Hospital, Second Military Medical University for her expertise in ELISA experiments.

\section{CONFLICTS OF INTEREST}

The authors have no conflicting interests.

\section{REFERENCES}

1. Villanueva A (2019) Hepatocellular carcinoma. N Engl J Med 380, 1450-1462

2. Hanahan D and Weinberg RA (2011). Hallmarks of Cancer: The Next Generation. Cell 144, 646-674

3. Cidon EU (2017) Systemic treatment of hepatocellular carcinoma: Past, present and future. World J Hepatol 9, 797-807

4. Chaffer CL and Weinberg RA (2011) A Perspective on Cancer Cell Metastasis. Science 331, 1559-1564

5. Tilghman SM (1985) The structure and regulation of the alpha-fetoprotein and albumin genes. Oxf Surv Eukaryot Genes 2, 160-206

6. Chen H, Egan JO and Chiu JF (1997) Regulation and activities of alpha-fetoprotein. Crit Rev Eukaryot Gene Expr 7, 11-41

7. Feuerman $\mathrm{MH}$, Godbout $\mathrm{R}$, Ingram RS and Tilghman $\mathrm{SM}$ (1989) Tissue-Specific Transcription of the Mouse AlphaFetoprotein Gene Promoter Is Dependent on Hnf-1. Mol Cell Biol 9, 4204-4212

8. Boisjoyeux B and Danan JL (1994) Members of the CAAT/ Enhancer-Binding Protein, Hepatocyte Nuclear Factor-I and Nuclear Factor-I Families Can Differentially Modulate the Activities of the Rat Alpha-Fetoprotein Promoter and Enhancer. Biochem J 301, 49-55

9. Xie Z, Zhang H, Tsai W et al (2008) Zinc finger protein ZBTB20 is a key repressor of alpha-fetoprotein gene transcription in liver. Proc Natl Acad Sci U S A 105, 10859-10864

10. Zhang H, Cao DM, Zhou LT et al (2015) ZBTB20 is a sequence-specific transcriptional repressor of alpha-fetoprotein gene. Sci Rep 5,11979

11. Shen H, Luan F, Liu H et al (2008) ZHX2 is a repressor of alpha-fetoprotein expression in human hepatoma cell lines. J Cell Mol Med 12, 2772-2780

12. Lee KC, Crowe AJ and Barton MC (1999) p53-mediated repression of alpha-fetoprotein gene expression by specific DNA binding. Mol Cell Biol 19, 1279-1288

13. Nguyen TT, Cho K, Stratton SA and Barton MC (2005) Transcription factor interactions and chromatin modifications associated with p53-mediated, developmental repression of the alpha-fetoprotein gene. Mol Cell Biol 25, 2147-2157
14. Chen Z, Yu L, Wu H et al (2003) Identification of a novel liver-specific expressed gene, TCP10L, encoding a human leucine zipper protein with transcription inhibition activity. J Hum Genet 48, 556-563

15. Zhong Z, Qiu J, Chen X et al (2008) Identification of TCP10L as primate-specific gene derived via segmental duplication and homodimerization of TCP10L through the leucine zipper motif. Mol Biol Rep 35, 171-178

16. Jiang DJ, Yu HX, Hexige SY et al (2004) Human liver specific transcriptional factor TCP10L binds to MAD4. J Biochem Mol Biol 37, 402-407

17. Zhong ZM, Qiu JP, Chen XY et al (2008) Identification of TCP10L as primate-specific gene derived via segmental duplication and homodimerization of TCP10L through the leucine zipper motif. Mol Biol Rep 35, 171-178

18. Yu HX, Jiang DJ, Guo ZK et al (2005) TCP10L is expressed specifically in spermatogenic cells and binds to death associated protein kinase-3. Int J Androl 28, 163-170

19. Shen SQ, Zuo J, Feng $\mathrm{H}$ et al (2016) TCP10L synergizes with MAD1 in transcriptional suppression and cell cycle arrest through mutual interaction. BMB Rep 49, 325-330

20. Zuo J, Cai H, Wu Y et al (2014) TCP10L acts as a tumor suppressor by inhibiting cell proliferation in hepatocellular carcinoma. Biochem Biophys Res Commun 446, 61-67

21. Chen Z, Yu L, Wu H et al (2003) Identification of a novel liver-specific expressed gene, TCP10L, encoding a human leucine zipper protein with transcription inhibition activity. J Hum Genet 48, 556-563

22. Johnson AD (1995) The Price of Repression. Cell 81, 655658

23. Gray $S$ and Levine $M$ (1996) Transcriptional repression in development. Curr Opin Cell Biol 8, 358-364

24. Biamonti G and Riva S (1994) New insights into the auxiliary domains of eukaryotic RNA binding proteins. FEBS Lett 340, 1-8

25. Leng X, Cooney AJ, Tsai SY and Tsai MJ (1996) Molecular mechanisms of COUP-TF-mediated transcriptional repression: Evidence for transrepression and active repression. Mol Cell Biol 16, 2332-2340

26. Peyton DK, Huang MC, Giglia MA, Hughes NK and Spear BT (2000) The alpha-fetoprotein promoter is the target of Afr1-mediated postnatal repression. Genomics 63, 173-180

27. Jin DK, Vacher J and Feuerman MH (1998) AlphaFetoprotein gene sequences mediating Afr2 regulation during liver regeneration. Proc Natl Acad Sci U S A 95, 8767-8772

28. Li MS, Li PF, He SP, Du GG and Li G (2000) The promoting molecular mechanism of alpha-fetoprotein on the growth of human hepatoma Bel7402 cell line. World J Gastroenterol 8, 469-475

29. Li MS, Li PF, Chen Q et al (2004) Alpha-fetoprotein stimulated the expression of some oncogenes in human hepatocellular carcinoma Bel 7402 cells. World J Gastroenterol 10, 819-824

30. Nakao K and Ichikawa T (2013) Recent topics on alphafetoprotein. Hepatol Res 43, 820-825 